\title{
Comparing lightwand-guided intubation techniques
}

\author{
Jian-Hua Liu ${ }^{1} \cdot$ Fu-Shan Xue ${ }^{1} \cdot$ Hui-Xian Li ${ }^{1}$
}

Received: 3 November 2016 / Accepted: 5 November 2016 / Published online: 11 November 2016

(C) Japanese Society of Anesthesiologists 2016

Keywords Lightwand · Tracheal intubation · Adjuvant techniques

\section{To the Editor:}

In a randomized clinical trial comparing single-handed chin lift and two-handed jaw thrust techniques for lightwandguided intubation in anesthetized patients, Yang et al. [1] showed that two-handed jaw thrust facilitated intubation and reduced the incidence and severity of postoperative sore throat (POST) compared to single-handed chin lift. However, in the Methods, the authors did not specify whether the two intubators were experienced in the lightwand-guided intubation, and whether they practiced the two techniques equally or preferred to use one of the techniques before this study. In fact, experience and competence with any intubation technique is critical for its successful use in any clinical setting [2]. For the results of a comparative study to be valid, the investigators must be equally proficient with each studied technique to avoid bias. We are concerned that different proficiency levels of the investigators with the two techniques would have contributed to their findings.

Furthermore, the incidence and severity of POST were used as the final end-points of performance. However, postoperative analgesia was not standardized in this study. The authors did not provide the type and dose of postoperative analgesics and the timing of their administration in relation to assessment of POST. In the absence of comparing postoperative analgesic medications, we argue that the secondary findings and subsequent conclusions should be interpreted with caution, as they may have been determined by incomplete methodology.

Finally, it should be emphasized that a shortcoming of the two-handed jaw thrust technique is the need for an additional assistant.

\section{Compliance with ethical standards}

Conflict of interest All authors have no financial support and potential conflicts of interest for this work.

\section{References}

1. Yang SM, Kim H, Chang JE, Min SW, Lee JM, Hwang JY. A comparison of single-handed chin lift and two-handed jaw thrust for tracheal intubation using a lightwand. J Anesth 2016. doi:10.1007/s00540-016-2276-0

2. Behringer EC, Kristensen MS. Evidence for benefit vs novelty in new intubation equipment. Anaesthesia. 2011;66(Suppl 2):57-64.
This comment refers to the article available at doi:10.1007/ s00540-016-2276-0.

Fu-Shan Xue xuefushan@aliyun.com

1 Department of Anesthesiology, Plastic Surgery Hospital, Chinese Academy of Medical Sciences, Peking Union Medical College, 33 Ba-Da-Chu Road, Shi-Jing-Shan District, Beijing 100144, People's Republic of China 